\title{
Aplicación de un Modelo de Balance Social en una Universidad Pública Chilena
}

\author{
Application of a Social Balance Model in a Chilean Public Uni- \\ versity
}

Roberto Campos Troncoso'; Miguel Bustamante Ubilla²

1 CIEC Centro de Investigación y Estudios Contables, Facultad de Economía y Negocios, Universidad de Talca 2 Facultad de Economía y Negocios, Universidad de Talca.

\section{Resumen}

El presente trabajo confirma la aplicación de un Balance de Responsabilidad Social en una Universidad Pública chilena, perteneciente al Consorcio de Universidades Estatales de Chile, CRUCH. Para ello metodológicamente se identificaron los grupos de variables relevantes que se relacionan con las instituciones de educación superior, seguido de las variables e indicadores que definen dichos grupos. Adicionalmente, se determinó la evolución, en dos años consecutivos de variables e indicadores, a fin de medir los avances o retrocesos de la responsabilidad social en la universidad pública seleccionada. Los resultados dieron cuenta que, efectivamente fue posible y se logró aplicar un Balance Social en una Universidad Pública, identificando 20 variables que evolucionan de un total de 27 definidas para su aplicación. El resultado de este Balance arrojó un saldo de contribución social de U.S. 46,0620, Unidades de Estándar de Satisfacción Social (UESS) confirmando que la Universidad contribuye aportando beneficios netos como expresión de su Responsabilidad Social.

Palabras clave: Balance Social; Responsabilidad Social Universitaria; Comportamiento ético; índice de responsabilidad Social.

\section{Abstract}

This work confirms the application of a Social Responsibility Balance in a Chilean Public University, belonging to the Consortium of Chilean State Universities, CRUCH. For this, methodologically, the groups of relevant variables that are related to higher education institutions were identified, followed by the variables and indicators that define these groups. Additionally, the evolution, in two consecutive years, of variables and indicators was determined in order to measure the progress or setbacks of social responsibility in the selected public university. The results showed that it was indeed possible and it was possible to apply a Social Balance in a Public University, identifying 20 variables that evolve from a total of 27 defined for its application. The result of this Balance returned a balance of U.S. social contribution. 46.0620, Standard Units of Social Satisfaction (UESS) confirming that the University contributes by providing net benefits as an expression of its Social Responsibility.

e-mail: mabu@utalca.c

Recibido: May 5, 2020 Aprobado: May 28, 2020 index. 


\section{INTRODUCCIÓN}

En la primera parte del artículo se presentan los basamentos teóricos, referidos a Responsabilidad Social (RS), presentando Conceptos de Responsabilidad Social; Desarrollo Sustentable y el Comportamiento Ético. En la segunda parte se lleva a cabo la propuesta del modelo de responsabilidad social para universidades RSU y su validación mediante la aplicación en una Universidad Pública chilena.

En referencia a los aspectos conceptuales, se afirma que "La Responsabilidad Social es comportamiento ético" (Bravo, Responsabilidad Social RS La nueva riqueza de las naciones, 2007, p. 25), así mismo se agrega que es “...una forma elevada de relacionarse con los demás, es crear sinergias que conduzcan a aumentar el bien común de corto y largo plazo en armonía con el entorno y los intereses comunes e individuales, tácitos y explícitos. También es practicar y fomentar el comportamiento ético, en especial, hacernos cargo de todas nuestras formas de influencia sobre las personas y el medio ambiente" (Bravo, Responsabilidad Social RS La nueva riqueza de las naciones, 2007, p. 26).

"La responsabilidad que las instituciones de educación superior tienen frente a la sociedad, proviene directamente de su misión social, en la cual se establecen las funciones principales de una universidad, las que abarcan ámbitos como la docencia, la investigación, la vinculación con el medio y la gestión universitaria” (Ganga Contreras \& Navarrete Andrade, 2012, p. 246). "La gran responsabilidad de convertir a los estudiantes en autoridades éticamente responsables, que tomen decisiones que afectaran el bien común. En materia de investigación, la universidad se hace responsable por la generación de conocimiento relevante y significativo; coherente con el desarrollo futuro de la sociedad” (Ganga Contreras \& Navarrete Andrade, 2012, p. 246).

Por lo tanto, se puede inferir que la Responsabilidad Social Universitaria tiene dos enfoques que son totalmente distinguibles. El primer enfoque mira hacia el interior del centro educativo, que debe ser metódico y para todos los eventos relacionados con los alumnos que permanecen en la Universidad, principalmente formando valores humanos, como la solidaridad, tolerancia, y principios de convivencia. Para formar conocimiento de su obligación personal y colectiva de ayudar a los que lo necesitan y mejorar su medio. El segundo enfoque es la mirada amplia y comprensiva hacia el exterior de la Universidad, esto es, hacia la sociedad en general, asumiendo que su labor, como instituciones de la sociedad, es también la de integrarse mediante acciones directas de intervención sobre el medio que las rodea, acoge y necesita (RSU, 2014)

En general respecto a la Responsabilidad Social Interna podemos señalar que la Responsabilidad Social, no se trata apenas de respetar a los derechos laborales, a normas internacionales como las de la OIT y de garantizar los derechos humanos. Si no que también el concepto de Trabajo Decente, propuesto por la OIT, por Juan Somavía (1999), que tiene, entre sus finalidades, establecer las características que debe tener una relación laboral, que cumpla con los estándares laborales internacionales, de manera que el trabajo se realice en condiciones de libertad, igualdad, seguridad y dignidad humana (Burgos Romero, 2014, p. 101).

Es por ello que el propósito de este trabajo, es elaborar una propuesta de un modelo de balance social para las Universidades Estatales Chilenas, pertenecientes al Consorcio de Universidades Estatales de Chile. En relación a los pasos de la metodología, primero se identifican las dimensiones o grupos relevantes que se relacionan con las instituciones de educación superior, seguido de la identificación de las variables e inmediatamente de los indicadores que definen dichas variable y grupos. Adicionalmente, y luego de un proceso más detallado de los datos, se determinan los coeficientes que permiten medir la importancia relativa de los grupos, variables e indicadores, que para los efectos de incorporarlos a un modelo definido en una totalidad, se porcentualizan en términos relativos los grupos, variables e indicadores. El paso final, consiste en determinar un índice de responsabilidad social universitaria, que posteriormente se valida, a través de la aplicación del modelo en una Universidad Pública chilena. 


\section{MÉTODO}

El presente trabajo es un estudio exploratorio no-experimental (Kinnear \& Taylor, 2005), para cuyo desarrollo fue necesario acudir tanto a fuentes primarias como secundarias. Principalmente se utilizó como fuente primaria la información entregada por el Consorcio de Universidades Estatales de Chile (CUECH), cuyos datos permitieron direccionar el modelo a ser propuesto. Por una parte, a partir de la información secundaria que se obtuvo dada la disponibilidad de textos, papers y trabajos de investigación en torno al tema los cuales sirvieron para respaldar el estudio.

\section{Etapas del trabajo de campo}

Se identificaron, en primera instancia, los diversos actores relacionados con las entidades universitarias y se determinaron las variables, indicadores e índices relevantes para cada factor identificado. Una vez determinados, se realizó la comparación de dichas variables e indicadores con el modelo de base teórica que mide la responsabilidad social. Identificados las variables e indicadores, se determinaron los coeficientes recalculados que conforman la propuesta. Finalmente, se llevó a efecto una prueba y validación del Modelo Propuesto con datos provenientes de la Universidad de Talca.

Uno de los primeros pasos realizados para llegar al modelo que habría de ser propuesto fue identificar agentes o actores que se relacionen con las instituciones universitarias. Para esto fue necesario acudir a la opinión de expertos, quienes poseen una vasta experiencia en responsabilidad social y aplicación de balances sociales. Según los estudios realizados por el CUECH (2016), los agentes relacionados con las universidades, de mayor relevancia son, el Gobierno, las Empresas, los Empleados y funcionarios, los Alumnos y, la Sociedad en su conjunto (RSU, 2014). Para efectos de este estudio, estos agentes fueron asociados a diferentes grupos:

El Grupo N.1 corresponde al Gobierno (G1), siendo éste de vital importancia para las Universidades, debido a que es la principal fuente de financiamiento, otorgando anualmente grandes sumas de dinero que ayudan al mejor desempeño de las instituciones educativas. Por otro lado, es este actor el que fija ciertas leyes y normas a las que están sometidas las Universidades del estado.

Las Empresas forman el Grupo N.2 (G2), estando estrechamente relacionadas a las Universidades, ya que también aportan de distintas formas, capital necesario para el desarrollo sostenible de las Universidades. Además, de poder ser apoyadas en distintos proyectos por las Universidades y también juegan un rol importante en la empleabilidad de los futuros egresados.

Los Empleados y Funcionarios conforman el Grupo N3 (G3), esto se debe a que éstos aportan conocimientos a través de sus investigaciones. Por otra parte, realizan publicaciones derivadas de sus estudios, lo que da un mayor prestigio a las instituciones y garantiza la calidad académica, además de formar un capital humano de alto nivel, el capital intangible.

Los Alumnos forman el Grupo № 4 (G4), esto se debe a que son éstos la razón de existencia de las Universidades y su principal objetivo como institución. Las Universidades deben otorgar a este grupo las condiciones necesarias para su desarrollo profesional, intelectual, recreativo y personal.

La Sociedad constituye el Grupo № 5 (G5), se enmarca principalmente en el aporte tanto cultural como intelectual que realizan las Universidades en beneficio a ésta.

\section{RESULTADOS}

Para efectos de determinar un Balance Social en una Universidad Pública, se escogió una Institución Universitaria Pública chilena considerando la una mayor posibilidad de acceso a la información requerida. En general, se logró analizar la variación de 20 de un total de 27 variables. 
Tabla 1. Variaciones de las Variables Relevantes de RSU

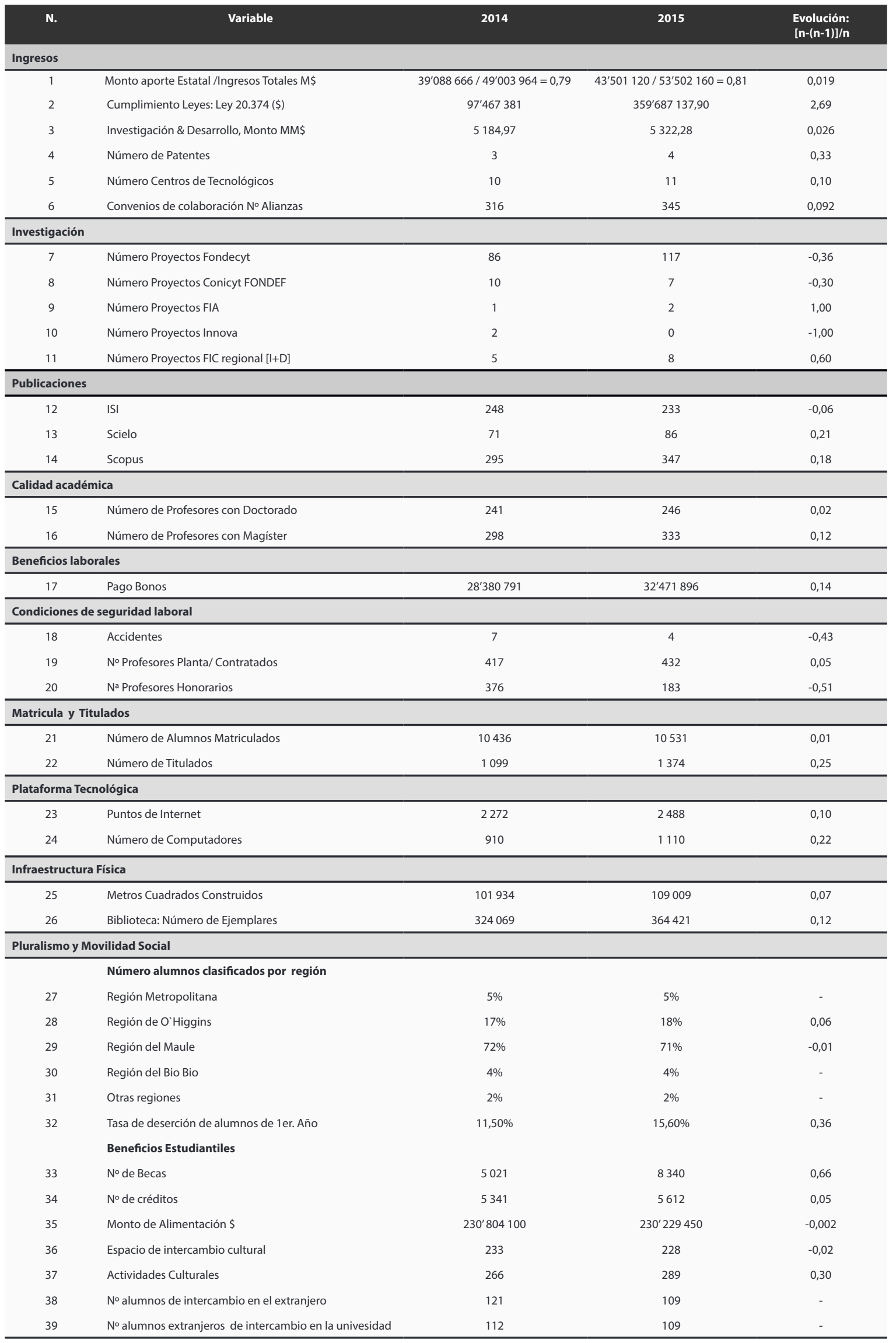




\begin{tabular}{|c|c|c|c|c|}
\hline \multicolumn{5}{|c|}{ Transparencia e información } \\
\hline 40 & Publicación de Balance Financiero & 1 & 1 & - \\
\hline 41 & Rentabilidad (Excedentes/ patrimonio) & $3,60 \%$ & $2,36 \%$ & $-0,35$ \\
\hline 42 & Liquidez ( AC / PC) & 1,97 & 2,32 & 0,35 \\
\hline 43 & Variación de Pasivos M\$ & $11^{\prime} 478481$ & $18^{\prime} 553938$ & 0,62 \\
\hline \multicolumn{5}{|c|}{ Beneficio Social } \\
\hline 44 & Generación de Anuarios & 2 & 2 & - \\
\hline
\end{tabular}

De acuerdo a los datos que efectivamente se lograron obtener, se pudo realizar modificaciones en ciertas variables, indicadores e índices, según correspondiera a la naturaleza de la variables que se buscada medir. Las modificaciones se explican a continuación. De esta manera se presenta la Tabla 1 con la evolución de las variables relevantes de RSU de la Universidad Pública analizada.

En el grupo gobierno, específicamente en la variable "cumplimiento de leyes", se tiene como indicador "Ley 20.374", cuyo índice propuesto es "casos de cumplimiento de ley", sin embargo de acuerdo a los datos que se obtuvieron, se modifica este índice por "costos de cumplimiento de la ley 20.374".

En la variable "Convenios de colaboración", cuyo indicador es " $\mathrm{N}^{\circ}$ de alianzas", se tiene en como índice el " $\mathrm{N}^{\circ}$ de convenios nacionales / total de convenios" y " $\mathrm{N}^{\circ}$ de convenios internacionales / total de convenios". Sin embargo, debido a que sólo se registran convenios internacionales según las fuentes de información, se modifica el índice contemplando sólo el $\mathrm{N}^{\circ}$ de convenios internacionales, sin la existencia de un ratio, mostrando así la variación existente de los convenios internacionales de un año a otro.

Para hacer posible el ejemplo de Balance Social Universitario, fue necesario agrupar los rangos de ingresos familiares de los alumnos en tres categorías, en la variable "Pluralismo/ Movilidad social", en donde se tiene como indicador el " $\mathrm{N}^{\circ}$ de alumnos clasificados por nivel de ingreso", cuyo índice es " $\mathrm{N}^{\circ}$ alumnos clasificados por nivel de ingreso (quintiles) / $\mathrm{N}^{\circ}$ total de alumnos".

En el grupo "Alumnos", específicamente en la variable "Pluralismo y movilidad social" se tiene como indicador "Tasa de deserción de alumnos" cuyo índice es el " $\mathrm{N}^{\circ}$ de alumnos desertores $/ \mathrm{n}^{\circ}$ total de alumnos", el cual fue modificado por " $\mathrm{N}^{\circ}$ de alumnos desertores en el primer año/ $n^{\circ}$ total de alumnos", con el fin de adaptarse a los datos brindados por el área de planificación de la Universidad de Talca. En la variable "Transparencia e información", perteneciente al grupo "Sociedad", se tiene como indicador la "Publicación de balances financieros" cuyo índice se define de la misma manera, sin embargo para efectos de la validación del modelo propuesto fue modificado por " $\mathrm{N}^{\circ}$ de publicaciones de balances financieros acumulados”.

\section{Aplicación del balance de responsabilidad social universitaria}

Con los índices corregidos de las 20 variables en cuestión, y con los datos ya dispuestos para la construcción del Balance, se debió calcular la variación porcentual de cada indicador, donde seguidamente, se debió calcular la evolución que experimentó cada variable del año 2014 al 2015 .

Para efectos de presentación del Balance, el inflactor asignado corresponde a un valor fijo 100, dado que su fin es número constante que sólo amplifica los valores. Los coeficientes corresponden a los de las variables; el factor es la multiplicación entre el inflactor y el coeficiente de las variables; y el total es el producto entre la evolución y el factor. Posteriormente, se decide según lo que indiquen las variables si ese número del total, sea negativo o positivo, corresponde a un activo o un pasivo para la organización.

La Tabla 2 el Balance de Responsabilidad Social R.S.U. determinado a modo de verificación de la factibilidad de determinar un balance Social. El resultado de este Balance arrojó un saldo de contribución social de U.S. 46,0620, unidades de satisfacción social (US) lo que significa que la Universidad Pública analizada, es efectivamente responsable en su accionar y contribuye, en términos netos con la sociedad a la cual sirve en los diferentes ámbito de actuación social. 
Tabla 2. Balance R.S.U. (2014-2015) de Universidad Pública

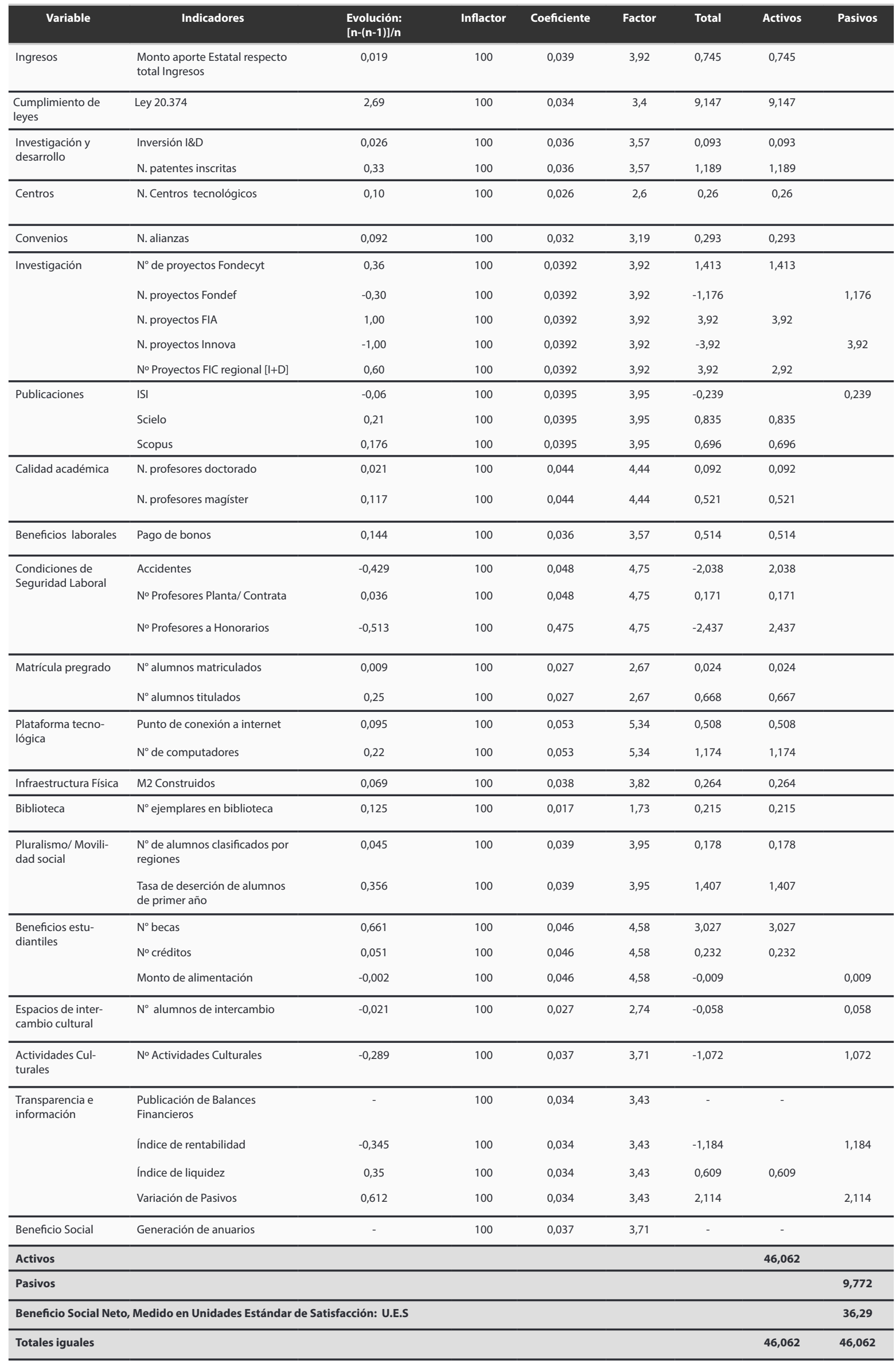




\section{DISCUSIONES}

La aplicación del balance social universitario permite conformar que la herramienta es factible, se demuestra que la RSU puede ser aplicada fácilmente una vez definidas las variables e indicadores (Bernal, 2010). Así en el caso de la Universidad Pública elegida para el presente estudio de la responsabilidad social y de acuerdo a los datos y mediciones obtenidas se pudo obtener un resultado positivo en la Ley 20.374 referida al monto de bonificación por retiro, lo que es bueno para la institución ya que implica que ésta evoluciona positivamente de un año a otro (Bravo, 2007), mostrando una disminución en los costos del cumplimiento de la mencionada ley. En general se aprecia que la variable convenios de colaboración evolucionó positivamente (CUECH, 2016), se logró efectivamente un resultado positivo, ya que esta institución aumentó sus convenios con diversas empresas (Burgos, 2014), lo que permite desarrollar aún más la experiencia como institución educativa y posicionarse en el mercado laboral, obteniendo un resultado en el balance de 0,293 positivos de unidades de responsabilidad social.

Por otra parte, los resultados muestran que respecto de Empleados - Funcionarios, existen dos variables que precisamente y conveniente para la sociedad arrojan resultados positivos (Ganga \& Navarrete, 2012). Así, la variación en la cantidad de sus publicaciones, de evidente impacto global en el sistema universitario (CUECH, 2016) lo cual demuestra la mayor eficiencia alcanzada por sus cuerpo docente (RSU, 2014), todo lo cual permite demostrar que la calidad académica basada en indicadores como la cantidad de profesores con doctorado y magister ascendió de un año a otro (CUECH, 2016), que correlaciona e incide también sobre la variable investigación que a largo plazo tiene un resultado positivo (Bernal, 2010).

Respecto de los beneficios estudiantiles, espacios de intercambio cultural y plataforma tecnológica, una vez más la universidad analizada logró resultados positivos (Bravo, 2007), destacándose esta última variable, la cual dentro del grupo, tiene una alta ponderación, teniendo un factor total de 1,681. Las variables matrícula de pre-grado y pluralismo - movilidad social, también muestran buenos resultados (CUECH, 2016).

Desde las perspectivas de la información, comunicación y transparencia, definidas como claves de la gestión universitaria (Ganga \& Navarrete, 2012) se confirma que efectivamente inciden sobre la Sociedad (RSU, 2014), lo cual alcanza alta importancia y trascendencia frente a la Sociedad y entrega un resultado positivo ya que la Universidad Pública analizada se ocupa de entregar y publicar sus estados financieros, además del la presentación de índices financieros relevantes de la gestión.

Finalmente, la Universidad pública analizada, es ciertamente social y culturalmente responsable (CUECH, 2016), debido a que el resultado final del Balance refleja un superávit de 46,062 unidades de Satisfacción aproximadamente y que son unidades de Responsabilidad Social.

\section{CONCLUSIONES}

Luego de la aplicación del balance social universitario, se concluye que éste puede ser aplicado fácilmente una vez definidas las variables e indicadores. En el grupo empresas, de acuerdo a la variable convenios de colaboración se confirma su eficacia como variable de RSE. Respecto al grupo Empleados - Funcionarios, se concluye que existen dos variables que arrojan resultados positivos. Así, la variación en la cantidad de sus publicaciones aumentó lo que demuestra una mayor eficiencia en su cuerpo docente, el cual incide sobre la comunidad académica además de estudiantil y sociedad en su conjunto por los efectos que genera.

En el grupo Alumnos, las variables, beneficios estudiantiles, espacios de intercambio cultural y plataforma tecnológica, obtuvieron resultados positivos agregando valor a los beneficios de responsabilidad social universitaria. Finalmente, la variable transparencia de información, perteneciente al grupo Sociedad, aporta socialmente, pudiéndose concluir en consecuencia que la Universidad Pública analizada en este trabajo, efectivamente es socialmente responsable y contribuye activamente y en forma positiva sobre el bienestar y bien común que, desde su rol le corresponde realizar. 


\section{REFERENCIAS}

Aguirre, N. (2005). Balance social; expresión responsable de la organización con fines sociales (Electronica ed.).

Álvarez, J. (2006). Balance Social 2004-2005 (Electronica ed.). Colombia: Universidad Cooperativa de Colombia.

Argandoña, A. (1998). La Teoría de los Stakeholders y el Bien Común. Barcelona, España: Universidad de Navarra.

Aristóteles. (2001). Ética (3 $3^{\underline{a}}$ edición ed.). (L. S.A., Ed., \& J. Leyva, Trad.) Madrid, España: Alba.

Benveniste, S. (2002). El alcance del conjunto del concepto de Responsabilidad Social Corporativa de acuerdo a los organismos internacionales promotores del tema (Electronica ed.). España.

Bernal, C. A. (2010). Metodología de la Investigación, Administración, economía, humanidades y ciencias sociales. (O. f. Palma, Ed.) Bogotá, Colombia: Pearson.

Bravo, J. (2013). gestión de Procesos, valorando la Práctica. Santiago, Chile: Evolución S.A.

Argandoña, A. (1998). La Teoría de los Stakeholders y el Bien Común. Barcelona, España: Universidad de Navarra.

Bravo, J. (2007). Responsabilidad Social RS La nueva riqueza de las naciones. Santiago, Chile: Evolución S.A.

Burgos Romero, C. (2014). La Responsabilidad Social Empresarial RSE en Chile: Causas de su tardia difusión. Revista Internacional de Relaciones Públicas , IV (7), 95-116.

Bustamante, M. (2003). Fundamentos de un modelo de balance de responsabilidad social empresarial. Revista científica do programa de Mestrado Académico em Administracao da Universidade do Vale do Itajai Univali, Brasil , X (2), 1-2.

Bustamante, M. (2003). Fundamento de un modelo de responsabilidad social. Alcance , 10 (2), 343-368.

Bustamante, M. (1996). Elaboración de un modelo de balance de responsabilidad empresarial para la empresa de la séptima región, Chile. Universidad de Deusto. San sebastian: Universidad de Deusto.

Bustamante, M. (1984). Aplicación del balance de responsabilidad social "medición de la calidad de vida en una empresa regional”. Panorama socioeconómico .

Cortina, A. (1996). Ética Empresarial. Madrid, España: Simáncas.

De Dios, T., \& Oubiña, J. (2012). Hombre, ética y responsabilidad social corporativa desde un enfoque antropológico. Madrid, España: Autónoma.

Del Mar, M. (2009). Propuesta de modelo de Responsabilidad Social Universitaria RSU para ser implementada. Asunción: Universidad Católica Nuestra señora de la asunción.

Estatales, C. d. (1 de Noviembre de 2016). uestatales.cl. Recuperado el 10 de Noviembre de 2016, de Consorcio de Universidades Estatales: www.uestatales.cl

Ethos, I. (2002). El balance social y la comunicación de la empresa con la sociedad (electronica ed.).

Fernández, J. (1999). Ética, Responsabilidad Social y Modelos de Empresas. Madrid, España: Universidad pontificia Comillas.

Freeman, R. E. (2010). A Stakeholders Approach. New York, Estados Unidos: Editorial Cambridge University Press.

Freeman, R. E. (1984). A Stakelholders Approach . Boston, Estados Unifos: Pitman.

Gaete, R. (2015). El concepto de Responsabilidad Social Universitaria desde la perspectiva de la alta dirección. Cuadernos de Administración,artículo de investigación científica y tecnológica, facultad de Ciencias de la Administración, Universidad del Valle, 31 (53), 97-107.

Ganga Contreras, F., \& Navarrete Andrade, E. (2012). Universidades privadas y su responsabilidad social en Chile: Un estudio exploratorio. opcion , 28 (68), 243-256.

Gil, N. (2007). Obtenido de www.wikilearning.com

González, T. (2006). La responsabilidad social de la empresa un buen negocio (electronica ed.)

Jimenez, M., De Ferrari, J., Del Piano, C., \& Andrade, L. (2006). Responsabilidad Social Universitaria, una manera de ser Universidad. Teoría y práctica en la experiencia Chilena. Santiago, Chile: Universidad construye País.

Kinnear, T., \& Tavlor, J. (2005). Investigación de Mercados. México D.F., México: Mc Graw - Hill.

Llano, C. (1997). Dilemas Éticos de la Empresa Contemporanea. Ciudad de México, México: Fondo de Cultura Económica.

Martínez, C. (2002). Lineamientos estratégicos de gestión en el proceso de vinculación universidad sector productivo. Universidad de Zulia. Zulia: Universidad de Zulia.

Melé, D. (1990). Informe Sobre la ëtica Empresarial. Boletin del circulo de empresarios, España.

Mori, E. (2002). Obtenido de www.pnud.cl

Murraga, A. (1995). Responsabilidad y Balance Social hoy en día: un reto para las cooperativas.

Pérez Domínguez, f. (2015). La responsabilidad Social Universitaria. Universidad de Huelva, Anton Menger. Consejo social Universidad de Huelva.

Prorrectoria, D. d. (2015). Anuario Estadístico Institucional 2015. Universidad de Talca, Prorrectoria. Talca: Universidad de Talca.

Prorrectoria, D. d. (2014). Anuario Estadístico Institucional 2014. Universidad de Talca, Prorrectoria. Talca: Universidad de Talca.

Ráfols, C. (2002). ¿ Ética en las empresas o empresas ëticas? Avanzando en una implementación contradictoria. (E. Electronica, Ed.) Obtenido de www.scribd.com

República, C. G. (2015). Financiamiento Fiscal a la Educación Superior. División análisis contable Área de empresas públicas y Universidades, Santiago-Chile.

República, C. G. (2014). Financiamiento fiscal a la Educación superior. División Análisis Contable Aréa de empresas Públicas y Universidades, Santiago-Chile.

RSU. (2014). Reporte de Sustentabuilidad. Universidad de Talca, Responsabilidad Social Universitaria RSU. Talca: Universidad de Talca.

Samper, I. (2002). La Ética en la Empresa: depende de mi. Barcelona, España: Universidad de Navarra.

Thomas, R. (2005). ethicscenter.org. Obtenido de www.ethicscenter.org

Vallaeys, F. (2007). La Responsabilidad Social Universitaria Cómo entenderla para quererla y practicarla. Lima, Perú: Artigas.

Valencia, R. (2009). Balance Social como la catapulta para dejar el infantilismo en la responsabilidad social corporativa (Electronica ed.). 